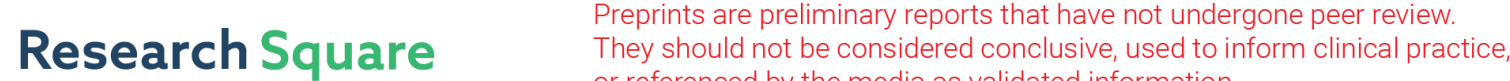 or referenced by the media as validated information. \\ Identification of Biomarkers Related To The Diagnosis And Prognosis of Thyroid Cancer Through Bioinformatics Analysis
}

\section{Xiao-Li Xie}

Fudan University Minhang Hospital

Hua-Li Yin

Central Hospital of Shaoyang

\section{Yu-Lin Pan}

Fudan University Minhang Hospital

\section{Guo-Xia Li}

Fudan University Minhang Hospital

\section{Chun-Yan Yuan}

Fudan University Minhang Hospital

Xia Sheng ( $\nabla$ shengxia_021@fudan.edu.cn )

Fudan University Minhang Hospital https://orcid.org/0000-0002-2137-7988

Primary research

Keywords: bioinformatics, biomarker, thyroid cancer, GEO, GSEA, TGCA

Posted Date: September 8th, 2021

DOI: https://doi.org/10.21203/rs.3.rs-740573/v1

License: (1) This work is licensed under a Creative Commons Attribution 4.0 International License. Read Full License 


\section{Abstract}

Background: Thyroid cancer is the most common malignant tumor of the head and neck. In recent years, the incidence of thyroid cancer (THCA) worldwide has rapidly increased and shows a trend in the younger generation. This study attempted to screen key genes and potential prognostic biomarkers for thyroid cancer using bioinformatics analysis.

Methods: This study attempted to screen key genes and potential prognostic biomarkers for thyroid cancer using bioinformatics analysis. 101 cases of thyroid cancer and 78 cases of normal thyroid tissue were collected from three Gene Expression Omnibus (GEO) databases, then we identified the differentially expressed genes (DEGs) and conducted downstream analyses. Moreover, we screened hub genes by constructing a protein-protein interaction (PPI) network. Finally, we assessed the expression level of hub genes in thyroid cancer tissue and its normal tissue using GEPIA and qRT-PCR respectively.

Results: 159 upregulated and 251 downregulated genes were determined after gene integration of these three GEO data sets. Through PPI analysis, we consider the top 20 DEGs with high connectivity as the hub genes of THCA. After that, this study verified 20 central genes through the GEPIA database and found that only four hub genes (TOP2A, FN1, TIMP1, and MMP9) had significantly higher expression levels in thyroid cancer tissues than in normal thyroid tissues. We further analyzed the correlation between these four hub genes and the prognosis of patients with thyroid cancer, which suggests that FN1, MMP9, TIMP1 help assess the prognosis of patients with thyroid cancer. We performed GSEA analysis on these 4 hub genes simultaneously, found that the high expression of these 4 hub genes enriched the "cell cycle." Subsequently, we collected thyroid cancer tissue specimens, verified these four hub gene expression levels by RT-PCR, and found that only FN1 and TIMP1 genes in thyroid cancer tissues had significantly higher mRNA levels than normal tissues.

Conclusions: Our research has identified 20 hub genes that may be related to the occurrence and development of thyroid cancer through multiple gene expression profile data sets and a series of comprehensive bioinformatics analyses. Further database and tissue validation analysis revealed that only 2 hub genes may be considered as potential prognostic biomarkers, including FN1 and TIMP1. In addition, these two hub genes are involved in the cell cycle, suggesting that they may play a role in the occurrence and development of thyroid cancer.

\section{Introduction}

Thyroid cancer is a malignant tumor originating from thyroid follicular epithelium or para-follicular epithelial cells, which is also the most common malignant tumor of the head and neck. In recent years, the incidence of thyroid cancer worldwide has rapidly increased and shows a trend in the younger generation. The incidence of thyroid cancer among women in urban areas in China ranks fourth among all malignant tumors in women, and it continues to increase at an annual rate of $20 \%$. Regarding the increase in the incidence of thyroid cancer year by year, some scholars have pointed out that the possible 
reasons are the increase in the number of patients willing to go to the hospital for cancer screening or treatment, the increase in the level of cancer detection, and the increase in the population exposed to radiation ${ }^{[1-2]}$. The prognosis of early thyroid cancer is generally good, but the prognosis of patients with advanced or recurrent metastatic thyroid cancer is poor ${ }^{[3]}$. Therefore, there is an urgent need to find reliable diagnostic and prognostic biomarkers to improve the therapeutic effect. At present, bioinformatics research based on high-throughput sequencing technology can comprehensively analyze changes in gene expression and has become an essential method for the study of cancer pathogenesis. This study intends to screen the transcriptome gene microarray expression profiles of thyroid cancer and normal tissues by searching the microarray data set in the GEO database and perform the functional enrichment analysis of differential genes, screen out the core genes with clinical significance through PPI analysis, and provide the basis for research on the mechanism of thyroid cancer.

This study involved 101 cases of thyroid cancer and 78 cases of normal thyroid tissue from 3 GEO data sets (GSE33630, GSE65144, and GSE29265). Through the analysis of 3 differential gene data sets, a total of 410 identical differentially expressed genes were screened out, including 159 up-regulated genes and 251 down-regulated genes in tumor samples. Through PPI analysis, we consider the top 20 DEGs with high connectivity as the hub genes of THCA. After that, this study verified 20 central genes through the GEPIA database and found that only four hub genes (TOP2A, FN1, TIMP1, and MMP9) had significantly higher expression levels in thyroid cancer tissues than in normal thyroid tissues. We further analyzed the correlation between these four hub genes and the prognosis of patients with thyroid cancer, which suggests that FN1, MMP9, TIMP1 help assess the prognosis of patients with thyroid cancer. We performed GSEA analysis on these 4 hub genes simultaneously, found that the high expression of these 4 hub genes enriched the "cell cycle." Subsequently, we collected thyroid cancer tissue specimens, verified these four hub gene expression levels by RT-PCR, and found that only FN1 (fibronectin 1) and TIMP1 (TIMP metallopeptidase inhibitor 1) genes in thyroid cancer tissues had significantly higher mRNA levels than normal tissues. Therefore, we speculate that FN1 and TIMP1 may be potential therapeutic targets and prognostic markers of thyroid cancer.

\section{Materials And Methods}

\subsection{Microarray data information}

The GEO (http://www.ncbi.nlm.nih.gov/geo/) database is a free public database of microarrays and is used for gene expression data sets and platform records. The gene expression profiles of GSE33630, GSE65144, and GSE29265 were chosen from the GEO database. GSE33630 was based on the GPL570 platform (HGU133_ Plus_2; Affymetrix Human Genome U133 Plus 2.0 Array), containing 11 anaplastic thyroid carcinomas (ATC), 49 papillary thyroid carcinomas (PTC), and 45 normal thyroids (N). GSE65144 was based on the GPL570 [HG-U133_Plus_2] Affymetrix Human Genome U133 Plus 2.0 Array, consisting of 12 ATC tissues and 13 normal thyroid tissues. The platform of GSE29265 was the GPL570 ([HGU133_Plus_2] Affymetrix Human Genome U133 Plus 2.0 Array) which included nine anaplastic thyroid 
carcinomas (ATCs), together with twenty papillary thyroid carcinomas (PTCs) paired with their respective adjacent tissues $(\mathrm{N})$. The data set information is shown in Table 1. The downloaded data was processed using the R package, then calibrated, standardized, and log2 transformation was performed on all data of gene expression.

Table 1

Details of the four Gene Expression Omnibus thyroid carcinoma data sets.

\begin{tabular}{|lllll|}
\hline GEO & Tissue & Platform & Normal & Tumor \\
\hline GSE33630 & thyroid & GPL570 & 45 & 60 \\
\hline GSE65144 & thyroid & GPL570 & 13 & 12 \\
\hline \multirow{2}{*}{ GSE29265 } & thyroid & GPL570 & 20 & 29 \\
\hline
\end{tabular}

\subsection{Screening for DEGs}

After downloading the 3 sets of original data sets, use R software for data processing, merging, standardization, and calculation of expression values. The data uses Fold-change and T-test for differential gene screening. The DEGs with $|\log \mathrm{FC}| \geq 1$ and adjust $P<0.05$ were considered as the cut-off criteria.

\subsection{GO term and KEGG pathway enrichment analysis of DEGs}

Gene Ontology (GO) is a biological model framework composed of three major parts: molecular function (MF), cell composition (CC), and biological process (BP). Jingdong Genome Encyclopedia (KEGG) is an information resource for understanding biological systems and genome functions from the molecular level. Use KOBAS3.0 (http://kobas.cbi.pku.edu.cn/kobas3/?t=1) for KEGG pathway analysis of differentially expressed genes, and use R package clusterProfiler for $\mathrm{GO}$ analysis.

\subsection{PPI network and module analysis}

The STRING database is an analytical database used to predict gene interaction networks in EMBL, which aggregates the extraction results from the Protein Interaction Network (PPI) database. Cytoscape's MCODE plug-in was used to evaluate the network model, and the genes in the top 3 templates were selected for pathway enrichment analysis. The results obtained through PPI were imported into the cytaHuba package of Cytoscape 3.8.2 software for analysis, and then the hub genes were screened according to the degree.

\subsection{Validation of the hub genes in GEPIA}

GEPIA (http://gepia.cancer-pku.cn/index.html) is a newly developed interactive web server that can analyze RNA from TCGA (The Cancer Genome Atlas) and GTEx (Genotype-Tissue Expression) databases Sequencing expression data. GEPIA provides differential expression analysis of tumor and normal 
tissues, analysis of the relationship between gene expression and pathological staging, analysis of patient survival, analysis of the correlation between genes, etc. In this study, GEPIA was used for hub gene expression analysis.

\subsection{Analyze the correlation between hub gene and pathological stage by GEPIAワas well as survival}

GEPIA was used to analyze the correlation between hub gene and pathological staging, as well as survival.

\subsection{GSEA analysis of hub gene}

GSEA (GSEA v2.0, available online: http://www.broad.mit.edu/gsea/) is used to analyze the biological pathways involved in the hub gene in thyroid cancer, and calculate the standardized enrichment score (NES), NOM p-val<0.05.

\subsection{Verify the expression of hub gene in thyroid cancer tissue by qPCR}

The expression level of the hub gene was verified by quantitative real-time PCR (qRT-PCR) in thyroid cancer tissues $(n=10)$ and surrounding normal tissues $(n=10)$.

\subsection{Statistical analysis}

The Kaplan-Meier (KM) curve was conducted to assess the association between the expression level of hub genes and the survival time of patients with thyroid cancer. The data were summarized by Excel and then statistically analyzed with GraphPad Prism5 (Graphpad Software, Inc, La Jolla, CA) software, and $P<0.05$ was considered as statistically significant.

\section{Results}

\subsection{Microarray data information and identification of DEGs in ATC and PTC}

Filter DEG through R software ( $|\log \mathrm{FC}| \geq 1$ and adjust $P<0.05$ ). In this study, 101 thyroid cancers and 78 normal thyroid tissues from 3 GEO data sets were involved. 1145 differential genes were screened from GSE33630; 2252 differential genes were screened by GSE65144; 792 differential genes were screened by GSE29265. Subsequently, 3 data sets of differential genes were analyzed, and a total of 410 identical differentially expressed genes were obtained, of which 159 genes were up-regulated in tumor samples and 251 genes were down-regulated. The DEGs of each gene expression profile data were visualized using a volcano plot (Figure 1). Subsequently, overlapping genes were screened in the thyroid cancer group compared with the control group using Venn software online (Figure 2).

\subsection{GO functional enrichment analysis}


To explore the biological functions of integrated DEG in ATC and PTC, we performed GO enrichment analysis on 410 DEGs (159 up-regulated genes and 251 down-regulated genes). The GO analysis results are divided into two parts. To explore the biological functions of integrated DEG in ATC and PTC, we performed GO enrichment analysis on 410 DEGs (159 up-regulated genes and 251 down-regulated genes). The GO analysis results are divided into two parts (up-regulated DEGs and down-regulated DEGs). In terms of biological processes, up-regulated DEGs are mainly abundant in the negative regulation of endoderm cell differentiation, endoderm formation, collagen fibril organization, endoderm development, and cell-matrix adhesion (Figure 3). In terms of cellular components, the upper DEGs mainly involve fibril collagen trimers, ribbon collagen fibrils, collagen trimer complexes, concentrated chromosomal microkinetochores, and extracellular matrix. In terms of molecular functions, the upward DEG mainly focuses on the combination of hyaluronic acid, extracellular matrix, proteoglycan, and the combination of extracellular matrix structural components that impart tensile strength and collagen. In the other part, in terms of biological processes, down-regulated DEGs are mainly abundant in the biological processes of thyroid hormone production, thyroid hormone metabolism, phenol-containing compound metabolism, hormone biosynthesis, and cold-induced thermogenesis (Figure 4). In terms of molecular functions, down-DEGs mainly focus on peroxidase activity, $\mathrm{SH} 3 / \mathrm{SH} 2$ adaptor activity, RNA polymerase II activation of transcription factor binding, oxidoreductase activity with peroxide as the receptor, and signal adaptor active. Using the ggplot2.R package, the bubble chart shows significant GO enrichment items, $P<0.05$.

\subsection{KEGG pathway analysis}

KEGG pathway analysis of the 159 up-DEGs was conducted in ATC and PTC using the KOBAS 3.0 online analysis tool, of which the top five (Figure 5) were "protein digestion and absorption," "p53 signaling pathway," "cytokine- cytokine receptor interaction," "ECM-receptor interaction," and "proteoglycans in cancer," respectively. The top five KEGG pathways in the 251 down-regulated DEGs (Figure 6) are "thyroid hormone synthesis," "metabolic pathways," "TGF-beta signaling pathways," "adipocytokine signaling pathways," and "regulation of lipolysis in adipocytes." The analysis indicated that the 410 dysregulated genes were mainly related to human cancer.

\subsection{PPI network construction, module analysis, and hub gene selection}

The PPI network was constructed for further investigation of the interaction among the integrated DEGs via Cytoscape software, based on the STRING database. The PPI network consisted of 376 nodes and 361 edges, among which including 159 upregulated and 251 downregulated genes (Figure 7). We regarded the top 20 DEGs with a high degree of connectivity as the hub genes of THCA (Table 2). The String database was used to construct an interactive network of all hit genes. Interestingly, all the hub genes formed a network of interactions (Figure 8).

\section{Table 2 Details of the 20 hub genes}




\begin{tabular}{|llll|}
\hline Gene & Degree & Type & MCODE Cluster \\
\hline CCNB2 & 20 & up & cluster1 \\
\hline TOP2A & 19 & up & cluster1 \\
\hline KIF20A & 19 & up & cluster1 \\
\hline NDC80 & 18 & up & cluster1 \\
\hline DLGAP5 & 18 & up & cluster1 \\
\hline NUSAP1 & 17 & up & cluster1 \\
\hline BUB1B & 17 & up & cluster1 \\
\hline ASPM & 17 & up & cluster1 \\
\hline CENPF & 16 & up & cluster1 \\
\hline RRM2 & 16 & up & cluster1 \\
\hline KIF4A & 16 & up & cluster1 \\
\hline CEP55 & 15 & up & cluster1 \\
\hline TPX2 & 15 & up & cluster1 \\
\hline PBK & 15 & up & cluster1 \\
\hline FN1 & 12 & up & cluster 2 \\
\hline KIF15 & 14 & up & cluster 2 \\
\hline MELK & 14 & up & cluster1 \\
\hline SDC2 & 14 & up & cluster1 \\
\hline TIMP1 & 13 & down & cluster 2 \\
\hline MMP9 & 12 & cluster1 \\
\hline
\end{tabular}

\subsection{Validation of the hub genes in GEPIA}

This study verified the expression level of hub genes in thyroid cancer tissue through GEPIA. The results showed that compared with normal thyroid tissues, TOP2A, FN1, TIMP1 and MMP9 were highly expressed in thyroid cancer tissues (Figure 9, $P<0.05$ ), while the differences in other genes were not significant. At the same time, the correlation between the four central genes was analyzed. The results showed that except for the uncorrelated relationship between TOP2A and FN1, the expression of other hub genes was significantly positively correlated (Figure 10, $P<0.05$ ). 
This study explored the relationship between the hub gene and Disease Free Survival (DFS) in patients with thyroid cancer. The results showed that, except for TIMP1, the DFS of the high expression group of TOP2A (DNA topoisomerase II alpha), FN1 and MMP9 were worse in the lower expression group (Figure $11, P<0.05)$. In addition, the study also found that in addition to TOP2A, the expression of the other three hub genes (FN1, MMP9, and TIMP1) were all related to the clinical stage of thyroid cancer (Figure 12, $P$ $<0.05)$.

\subsection{GSEA analysis of hub genes}

To further evaluate the hub genes, we used GSEA to analyze the biological pathways involved in the hub gene. We divided the patients between the data set into high expression groups and low expression groups according to the median of TOP2A, FN1, TIMP1, and MMP9. The results showed that the high expression of these three hub genes (FN1, TOP2A, MMP9) enriched the "cell cycle" (Figure 13, NOM $p$ val<0.05).

\section{8 qRT-PCR verifies the expression level of hub gene in thyroid cancer tissue}

We performed RT-PCR verification on the collected thyroid cancer tissues and surrounding normal tissues. The results found that the mRNA level of FN1 and TIMP1 genes in thyroid cancer tissues was significantly higher than that of normal tissues $(P<0.05)$, while TOP2A and MMP9 did not find significant differences (Figure 14).

\section{Discussion}

Thyroid cancer is prevalent. The incidence of thyroid cancer is on the rise worldwide, and the number of cases has increased sharply in the past decade ${ }^{[4]}$. Most oncologists support the use of next-generation sequencing to find potentially actionable mutations ${ }^{[5-6]}$. Although these mutations are small, treatments that target these targets are generally easier to tolerate and may produce longer-lasting responses. BRAF V600E is the most common molecular change in PTC. It is highly specific for PTC and exists in $36 \%$ to $80 \%$ of papillary carcinomas ${ }^{[7]}$. Researchers found that BRAF-mutated thyroid cancer has a higher lymph

node metastasis and recurrence rate after resection, and radioactive iodine therapy is ineffective ${ }^{[8]}$. Our results provide more treatment options for patients and perhaps improves the quality of life of patients. Bioinformatics combined with high-throughput sequencing can systematically analyze disease-related genes and is an important method for studying the pathogenesis of diseases.

In this study, 101 thyroid cancers and 78 normal thyroid tissues from 3 GEO data sets were involved. 1145 differential genes were screened from GSE33630; 2252 differential genes were screened by GSE65144; 792 differential genes were screened by GSE29265. Subsequently, 3 data sets of differential genes were analyzed, and a total of 410 identical differentially expressed genes were obtained, of which 159 genes were up-regulated in tumor samples and 251 genes were down-regulated. To reduce the error, the 3 GEO data sets we selected are all from the GPL570 platform. Through PPI analysis, we consider the top 20 DEGs with high connectivity as the hub genes of THCA. After that, this study verified 20 hub genes 
through the GEPIA database and found that only 4 hub genes (TOP2A, FN1, TIMP1, and MMP9) had significantly higher expression levels in thyroid cancer tissues than in normal thyroid tissues. We further analyzed the correlation between these 4 hub genes and the prognosis of patients with thyroid cancer, and the results showed that in addition to TIMP1, the DFS of the high expression group of TOP2A, FN1, and MMP9 were all lacking in the low expression group. In addition, the study also found that in addition to TOP2A, the expression of the other three hub genes (FN1, MMP9, and TIMP1) are all related to the clinical stage of thyroid cancer $(P<0.05)$, which further suggests that FN1, MMP9, and TIMP1 are useful for evaluation. The prognosis of thyroid cancer patients is helpful. We performed GSEA analysis on these 4 hub genes (TOP2A, FN1, TIMP1, and MMP9) at the same time, and found that the high expression of these 4 hub genes enriched the "cell cycle." Subsequently, we collected thyroid cancer tissue specimens and verified the expression of these four hub genes by RT-PCR, and found that only FN1 and TIMP1 genes in thyroid cancer tissues had significantly higher mRNA levels than normal tissues. Therefore, we speculate that FN1 and TIMP1 may be potential therapeutic targets and prognostic markers of thyroid cancer.

FN1 is a member of the fibronectin (FN) family of interstitial markers. FN1 signal activation has been confirmed to be closely related to EMT to promote tumor invasion and metastasis ${ }^{[9]}$. It is reported in the literature that FN1 is up-regulated in breast cancer, ovarian cancer, gastric cancer, and other human tumors ${ }^{[10-12]}$. Similarly in PTC, FN1 is more highly expressed in cancerous tissues than in adjacent tissues. FN1 knockout inhibits PTC cell proliferation, adhesion, and metastasis ${ }^{[13]}$. Some researchers pointed out that microRNA-139 can target FN1 to hinder PTC progress ${ }^{[14]}$. Studies have found through bioinformatics analysis that FN1 can be used as a prognostic marker for sporadic medullary thyroid carcinoma ${ }^{[15]}$. We found through bioinformatics that FN1 is highly expressed in thyroid cancer tissues and is related to the DFS of thyroid cancer, indicating that it is associated with the prognosis of thyroid cancer.

Tissue inhibitor matrix metalloproteinase 1 (TIMP1) belongs to the tissue inhibitor of the metalloproteinase family. Researchers found that by inducing TIMP1 to specifically regulate the FAKPI3K/AKT and MAPK pathways, inhibiting TIMP1 expression can reduce proliferation and metastasis, and increase cell apoptosis, which can be used a prognostic indicator of colon cancer ${ }^{[16]}$. Inhibition of TIMP1 expression can make pancreatic cancer sensitive to radiotherapy and chemotherapy ${ }^{[17]}$. Studies have pointed out that TIMP-1 can activate YAP/TAZ (Yes-related protein/transcriptional co-activator with PDZ binding motif) as a new downstream target to promote cell proliferation ${ }^{[18]}$. Furthermore, TIMP1 can promote cell proliferation in ovarian cancer, revealing TIMP1 as a potential therapeutic target for ovarian cancer ${ }^{[19]}$. Our results suggest that TIMP1 is highly expressed in thyroid cancer tissues and is related to the DFS of thyroid cancer, suggesting that it is related to the prognosis of thyroid cancer.

MMP-9 (matrix metallopeptidase 9) is a zinc-containing protease, which is abnormally expressed in a variety of tumors. Studies have found that overexpression of MMP-9 contributes to the aggressiveness of the prostate cancer cell line LNCaP ${ }^{[20]}$. In addition, active MMP-9 is significantly related to extrathyroid 
invasion, lymph node metastasis, and tumor invasion ${ }^{[21]}$. In vitro studies of MMP-9 overexpression showed that Notch-1 activation was increased, while $\beta$-catenin was correspondingly decreased, indicating that MMP-9 plays a tumor suppressor role in inflammatory bowel cancer, leading to p21 (WAF1/Cip1) activation and $\beta$-catenin inhibition ${ }^{[22]}$. Researchers found that the expression level of MMP-9 in thyroid cancer cells was higher than that of normal human thyroid cells, and by observing the changes in the biological behavior of thyroid cancer cell lines after interfering with MMP-9 expression, they found that MMP-9 can promote TGF- $\beta 1$ induction The EMT process of thyroid cancer affects the migration and invasion ability of cells ${ }^{[23]}$. Our results also suggest that MMP-9 is highly expressed in thyroid cancer tissues and is related to disease-free survival and clinical stage.

Topoisomerase lla (TOP2A) is involved in DNA replication, transcription, recombination, and chromatin reconstruction. The enzyme maintains the topological state of chromosomes during DNA replication and transcription, catalyzes the instantaneous break and reconnection of two double-helical DNA strands, thereby changing DNA and topological structure, and controlling cell cycle progression. TOP2A is abnormally expressed in various tumors ${ }^{[24-26]}$ and is used in cancer treatment ${ }^{[27-30]}$. Double-strand break (DSB) is the most common type of genetic toxicity of DNA damage, and a single unrepaired DSB can trigger cell apoptosis. TOP2A can cause spontaneous DSB of genomic DNA ${ }^{[31]}$. The accumulation of DSB can kill tumor cells, but it may also cause genome instability. Studies have screened TOP2A as the hub gene of thyroid cancer through bioinformatics ${ }^{[22-34]}$. Our bioinformatics analysis results also indicate that TOP2A is highly expressed in thyroid cancer and is related to the prognosis, but the histological verification has not obtained the same results. To ensure the reproducibility of the results, a large number of tissue samples and a series of Cell experiments to verify this conclusion.

In summary, our research has identified 20 hub genes that may be related to the occurrence and development of thyroid cancer through multiple gene expression profile data sets and a series of comprehensive bioinformatics analyses. Further database and tissue validation analysis revealed that only 2 hub genes may be considered as potential prognostic biomarkers, including FN1 and TIMP1. In addition, these two hub genes are involved in the cell cycle, suggesting that they may play a role in the occurrence and development of thyroid cancer. However, in the future, a series of molecular biology experiments and a large number of clinical specimens are needed to verify these hub genes to provide a basis for the treatment and prognosis of thyroid cancer.

\section{Abbreviations}

GEO: Gene Expression Omnibus; DEGs: differentially expressed genes; PPI: protein-protein interaction; THCA: thyroid cancer; TOP2A: DNA topoisomerase II alpha; FN1: fibronectin 1; TIMP1: Tissue inhibitor matrix metalloproteinase 1; ATC: anaplastic thyroid carcinomas; PTC: papillary thyroid carcinomas; GO: Gene Ontology; MF: molecular function; CC: cell composition; BP: biological process; DFS: Disease Free Survival; MMP9: matrix metallopeptidase 9. 


\section{Declarations}

\section{Ethics approval and consent to participate}

This study was approved by the Ethics Committee of Fudan University Minhang Hospital.

\section{Consent for publication}

All recruited patients provided informed consent.

\section{Availability of data and materials}

All data generated or analyzed during this study are included in this published article. Further details are available from the corresponding author upon request.

\section{Competing interests}

The authors declare that the research was conducted in the absence of any commercial or financial relationships that could be construed as a potential conflict of interest.

\section{Funding}

This work was funded by the Specialized Medical Specialty Project in Minhang District, Shanghai (No. 2020MWFC03).

Author contributions XLX and HLY performed the experiments and wrote the manuscript supervised by YLP, GXL, CYY and XS who edited the manuscript. All authors read and approved the final manuscript.

\section{Acknowledgements}

Not applicable.

\section{References}

[1] Pellegriti G, Frasca F, Regalbuto C, et al. Worldwide increasing incidence of thyroid cancer: update on epidemiology and risk factors. J Cancer Epidemiol. 2013;2013:965212.

[2] Cabanillas ME, McFadden DG, Durante C. Thyroid cancer. Lancet. 2016; 388(10061):2783-2795.

[3] Chinese Society of Clinical Oncology (CSCO) diagnosis and treatment guidelines for persistent/recurrent and metastatic differentiated thyroid cancer 2018 (English version). Chin J Cancer Res. 2019;31(1):99-116.

[4] Li M, Dal Maso L, Vaccarella S. Global trends in thyroid cancer incidence and the impact of overdiagnosis. Lancet Diabetes Endocrinol. 2020;8(6):468-470. 
[5] Nikiforova MN, Wald Al, Roy S, Durso MB, Nikiforov YE. Targeted next-generation sequencing panel (ThyroSeq) for detection of mutations in thyroid cancer. J Clin Endocrinol Metab. 2013;98(11):E1852E1860.

[6] Cha YJ, Koo JS. Next-generation sequencing in thyroid cancer. J Transl Med. 2016;14(1):322.

[7] Tavares C, Melo M, Cameselle-Teijeiro JM, Soares P, Sobrinho-Simões M. ENDOCRINE TUMOURS: Genetic predictors of thyroid cancer outcome. Eur J Endocrinol. 2016;174(4):R117-R126.

[8] Xing M, Westra WH, Tufano RP, et al. BRAF mutation predicts a poorer clinical prognosis for papillary thyroid cancer. J Clin Endocrinol Metab. 2005;90(12):6373-6379.

[9] Park J, Schwarzbauer JE. Mammary epithelial cell interactions with fibronectin stimulate epithelialmesenchymal transition. Oncogene. 2014;33(13):1649-1657.

[10] Moon PG, Lee JE, Cho YE, et al. Fibronectin on circulating extracellular vesicles as a liquid biopsy to detect breast cancer. Oncotarget. 2016;7(26):40189-40199.

[11] Yousif NG. Fibronectin promotes migration and invasion of ovarian cancer cells through upregulation of FAK-PI3K/Akt pathway. Cell Biol Int. 2014;38(1):85-91.

[12] Sun Y, Zhao C, Ye Y, et al. High expression of fibronectin 1 indicates poor prognosis in gastric cancer. Oncol Lett. 2020;19(1):93-102.

[13] Xia S, Wang C, Postma EL, Yang Y, Ni X, Zhan W. Fibronectin 1 promotes migration and invasion of papillary thyroid cancer and predicts papillary thyroid cancer lymph node metastasis [published correction appears in Onco Targets Ther. 2017 May 29;10:2737-2738]. Onco Targets Ther. 2017;10:17431755.

[14] Ye Y, Zhuang J, Wang G, He S, Ni J, Xia W. MicroRNA-139 targets fibronectin 1 to inhibit papillary thyroid carcinoma progression. Oncol Lett. 2017;14(6):7799-7806.

[15] Zhan S, Li J, Wang T, Ge W. Quantitative Proteomics Analysis of Sporadic Medullary Thyroid Cancer Reveals FN1 as a Potential Novel Candidate Prognostic Biomarker. Oncologist. 2018;23(12):1415-1425.

[16] Song G, Xu S, Zhang H, et al. TIMP1 is a prognostic marker for the progression and metastasis of colon cancer through FAK-PI3K/AKT and MAPK pathway. J Exp Clin Cancer Res. 2016;35(1):148.

[17] D'Costa Z, Jones K, Azad A, et al. Gemcitabine-Induced TIMP1 Attenuates Therapy Response and Promotes Tumor Growth and Liver Metastasis in Pancreatic Cancer. Cancer Res. 2017;77(21):5952-5962.

[18] Ando T, Charindra D, Shrestha M, et al. Tissue inhibitor of metalloproteinase-1 promotes cell proliferation through YAP/TAZ activation in cancer. Oncogene. 2018;37(2):263-270. 
[19] Abreu M, Cabezas-Sainz P, Alonso-Alconada L, et al. Circulating Tumor Cells Characterization Revealed TIMP1 as a Potential Therapeutic Target in Ovarian Cancer. Cells. 2020;9(5):1218.

[20] Aalinkeel R, Nair BB, Reynolds JL, et al. Overexpression of MMP-9 contributes to invasiveness of prostate cancer cell line LNCaP. Immunol Invest. 2011;40(5):447-464.

[21] Marecko I, Cvejic D, Selemetjev S, et al. Enhanced activation of matrix metalloproteinase-9 correlates with the degree of papillary thyroid carcinoma infiltration. Croat Med J. 2014;55(2):128-137.

[22] Garg P, Sarma D, Jeppsson S, et al. Matrix metalloproteinase-9 functions as a tumor suppressor in colitis-associated cancer. Cancer Res. 2010;70(2):792-801.

[23] Li Y, He J, Wang F, et al. Role of MMP-9 in epithelial-mesenchymal transition of thyroid cancer. World J Surg Oncol. 2020;18(1):181.

[24] Panvichian R, Tantiwetrueangdet A, Angkathunyakul N, Leelaudomlipi S. TOP2A amplification and overexpression in hepatocellular carcinoma tissues. Biomed Res Int. 2015;2015:381602.

[25] Du X, Xue Z, Lv J, Wang H. Expression of the Topoisomerase II Alpha (TOP2A) Gene in Lung Adenocarcinoma Cells and the Association with Patient Outcomes. Med Sci Monit. 2020;26:e929120.

[26] Huijsmans CJ, van den Brule AJ, Rigter H, et al. Allelic imbalance at the HER2/TOP2A locus in breast cancer. Diagn Pathol. 2015;10:56.

[27] Ghisoni E, Maggiorotto F, Borella F, et al. TOP2A as marker of response to pegylated lyposomal doxorubicin (PLD) in epithelial ovarian cancers. J Ovarian Res. 2019;12(1):17.

[28] Ito F, Furukawa N, Nakai T. Evaluation of TOP2A as a Predictive Marker for Endometrial Cancer With Taxane-Containing Adjuvant Chemotherapy. Int J Gynecol Cancer. 2016;26(2):325-330.

[29] Ejlertsen B, Jensen MB, Nielsen KV, et al. HER2, TOP2A, and TIMP-1 and responsiveness to adjuvant anthracycline-containing chemotherapy in high-risk breast cancer patients. J Clin Oncol. 2010;28(6):984990.

[30] Nielsen KV, Ejlertsen B, Møller S, et al. The value of TOP2A gene copy number variation as a biomarker in breast cancer: Update of DBCG trial 89D. Acta Oncol. 2008;47(4):725-734.

[31] Morimoto S, Tsuda M, Bunch H, et al. Type II DNA Topoisomerases Cause Spontaneous DoubleStrand Breaks in Genomic DNA. Genes (Basel). 2019;10(11):868.

[32] Li L, Zhu M, Huang H, Wu J, Meng D. Identification of Hub Genes in Anaplastic Thyroid Carcinoma: Evidence From Bioinformatics Analysis. Technol Cancer Res Treat. 2020;19:1533033820962135.

[33] Hu S, Liao Y, Chen L. Identification of Key Pathways and Genes in Anaplastic Thyroid Carcinoma via Integrated Bioinformatics Analysis. Med Sci Monit. 2018 Sep 14;24:6438-6448. 
[34] Gao X, Wang J, Zhang S. Integrated Bioinformatics Analysis of Hub Genes and Pathways in Anaplastic Thyroid Carcinomas. Int J Endocrinol. 2019;2019:9651380.

\section{Figures}

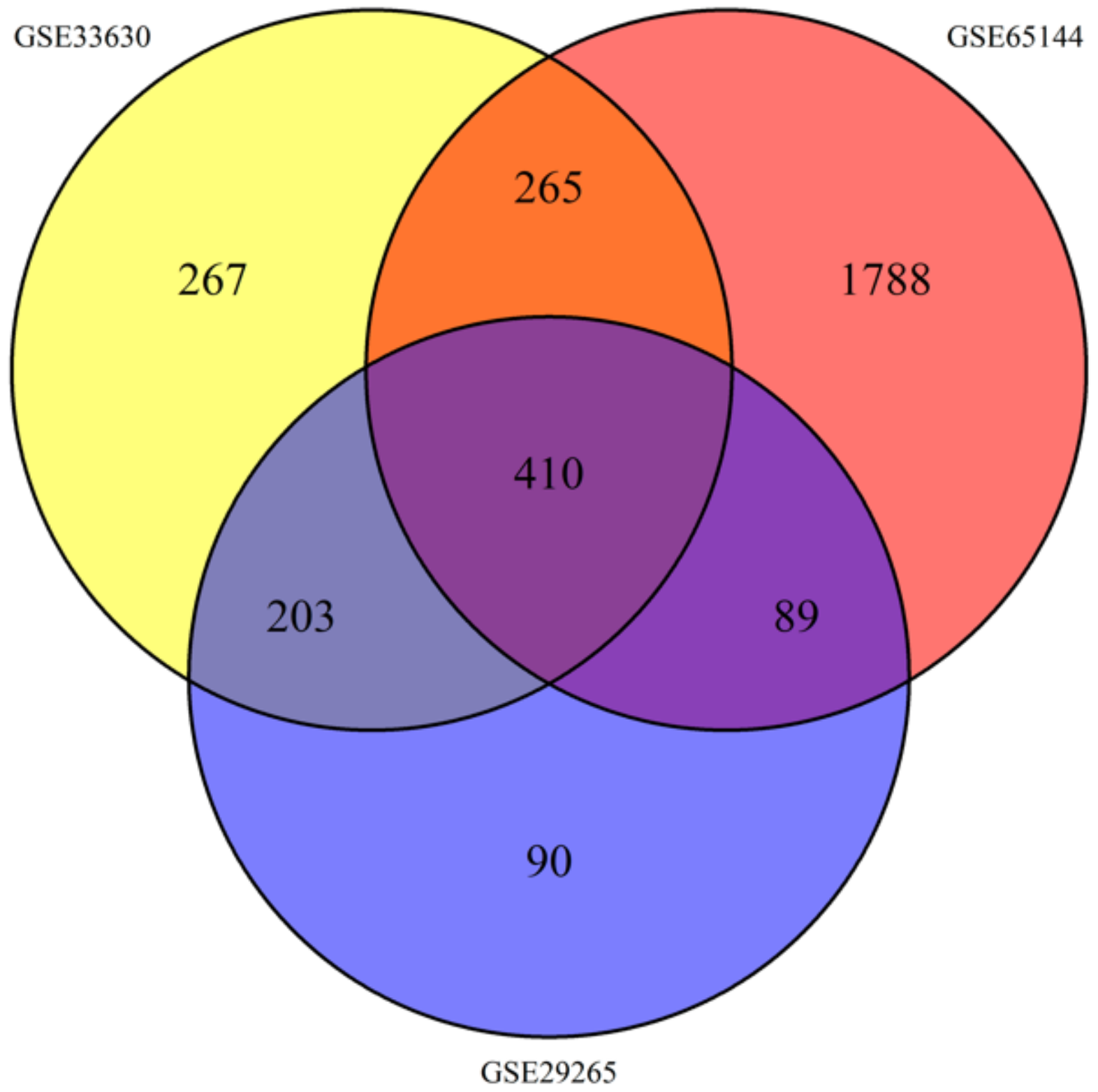

Figure 1

Venn plots of overlapping DEGs. 


\section{Tumoral vs Normal \\ Cutoff for $\log \mathrm{FC}$ is 1}

The number of up gene is 159

The number of down gene is 251

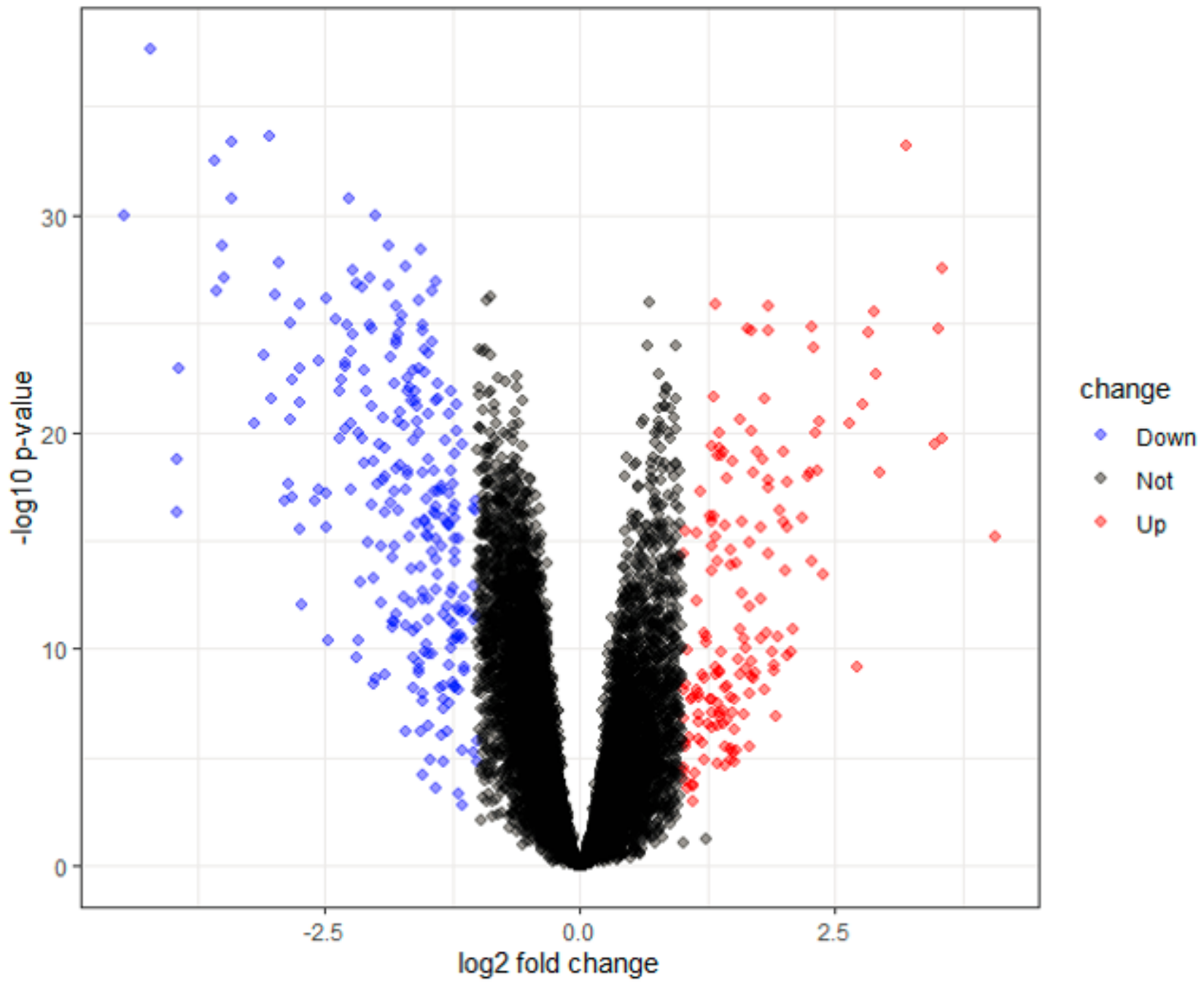

Figure 2

Volcano plots of gene expression profile data in GSE33630, GSE65144 and GSE29265. 
GO Enriched(upgene-top10)

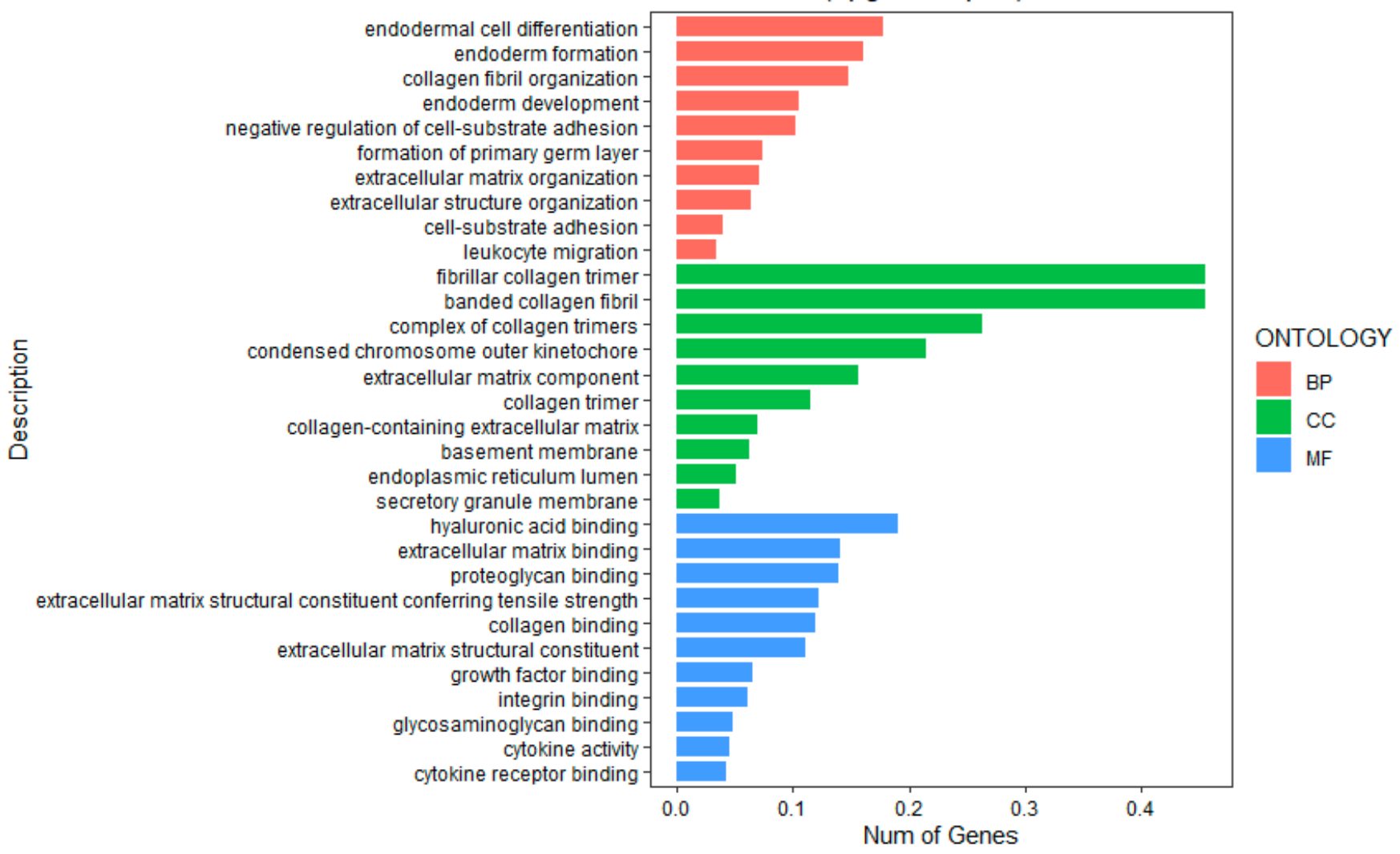

Figure 3

GO analysis of the upregulated DEGs. 


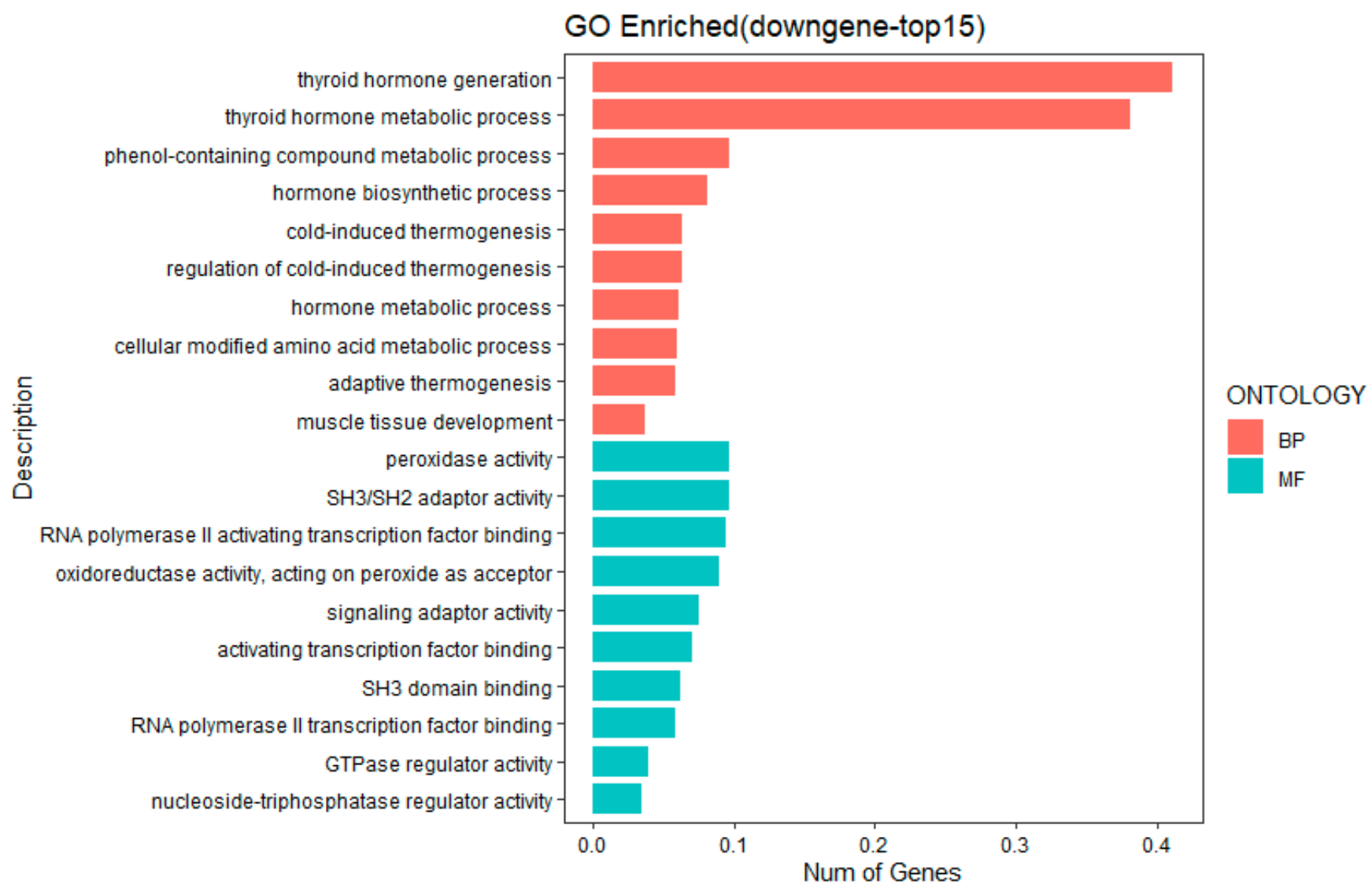

Figure 4

GO analysis of the downregulated DEGs. 


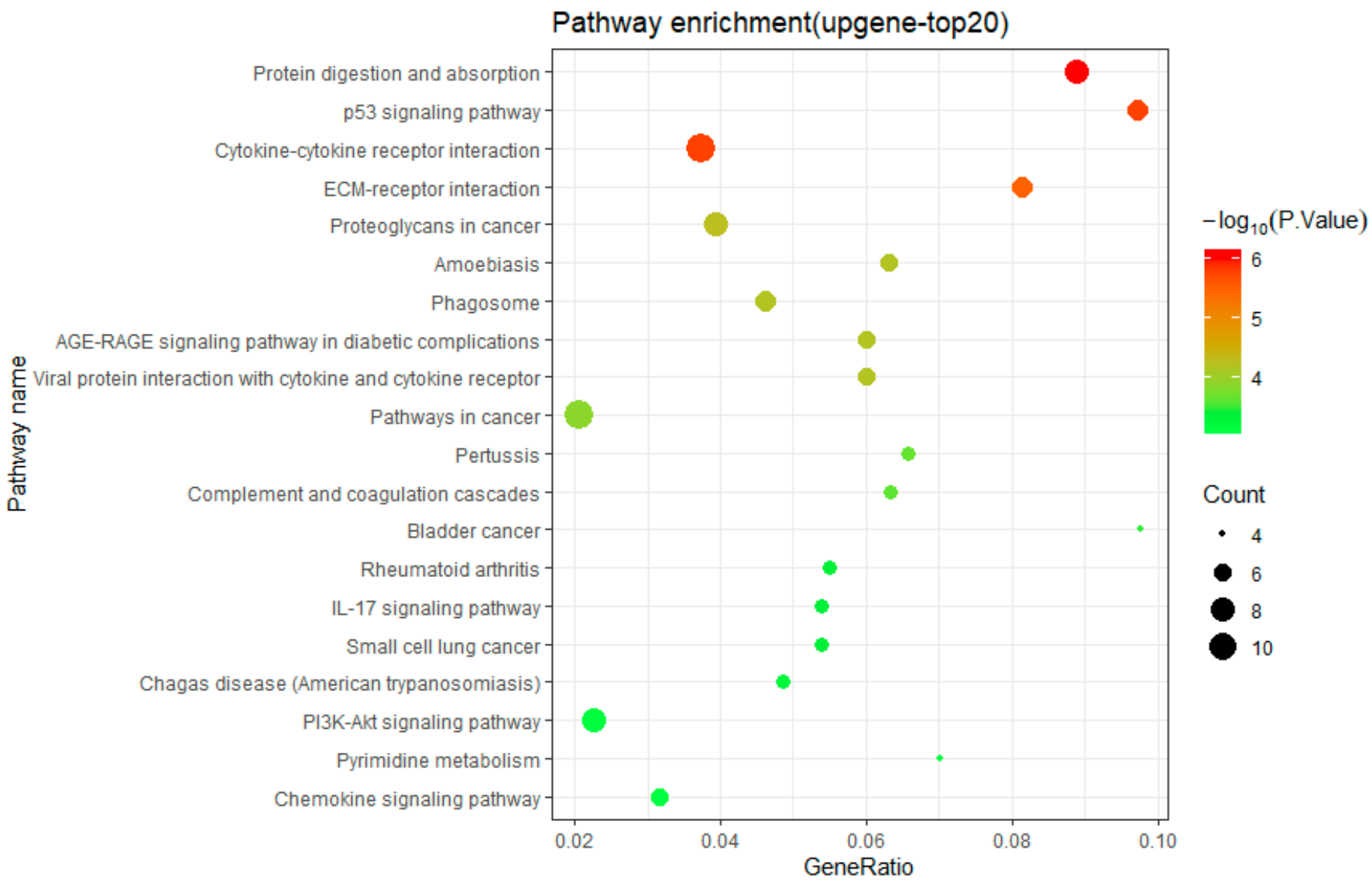

Figure 5

KEGG analysis of the upregulated DEGs 


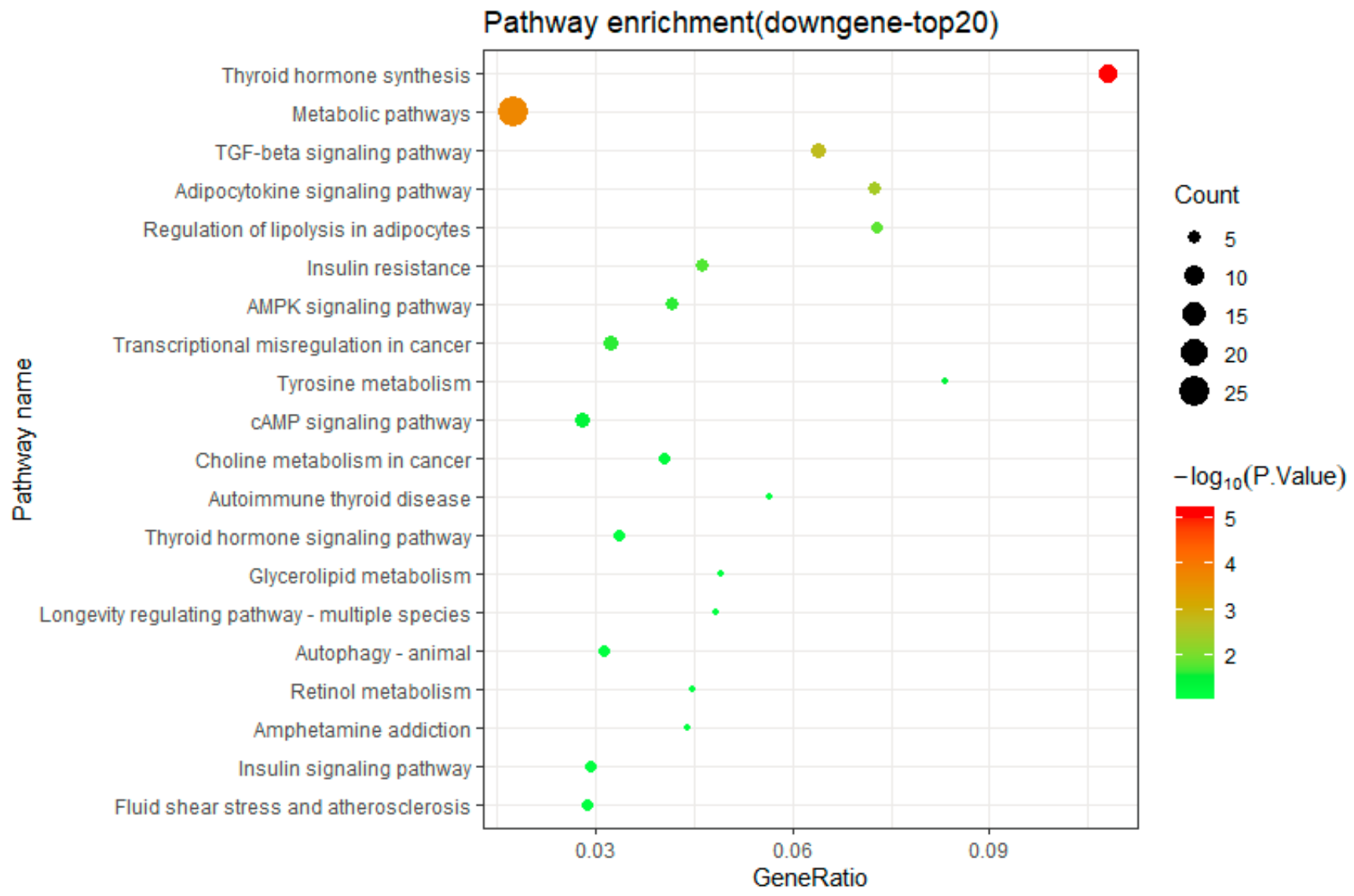

Figure 6

KEGG analysis of the downregulated DEGs 
(A)

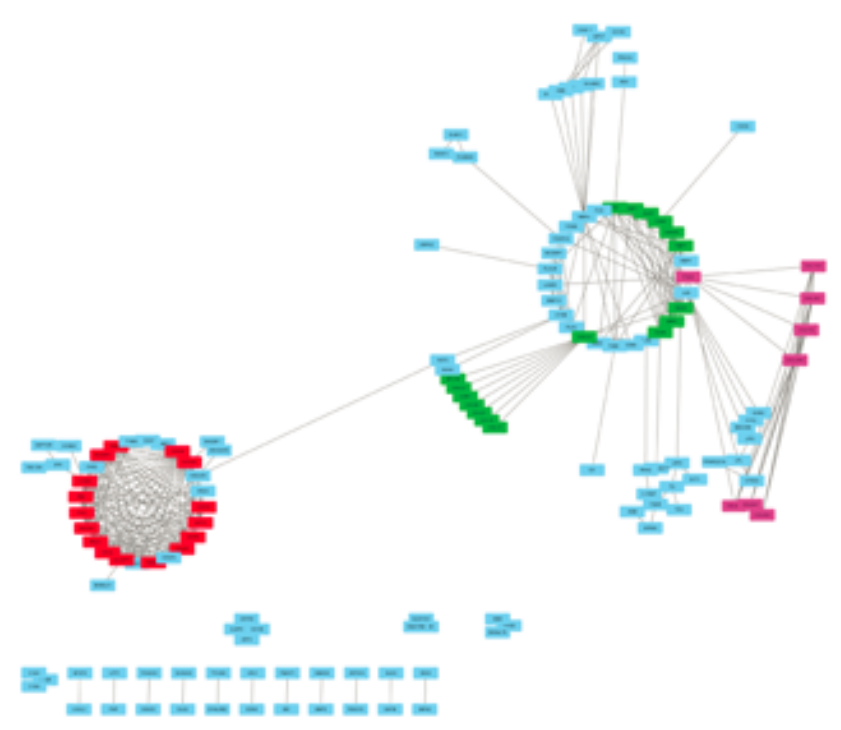

(C)

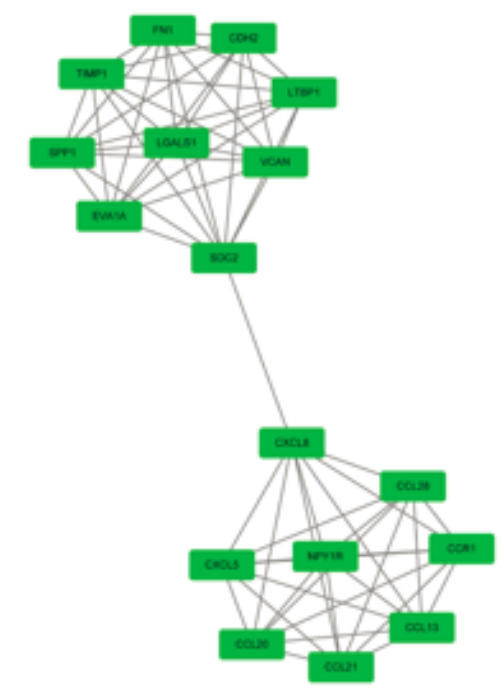

(B)

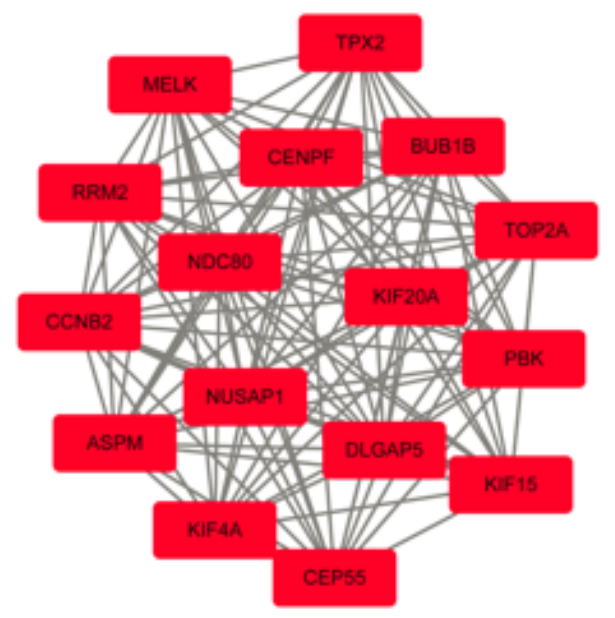

(D)

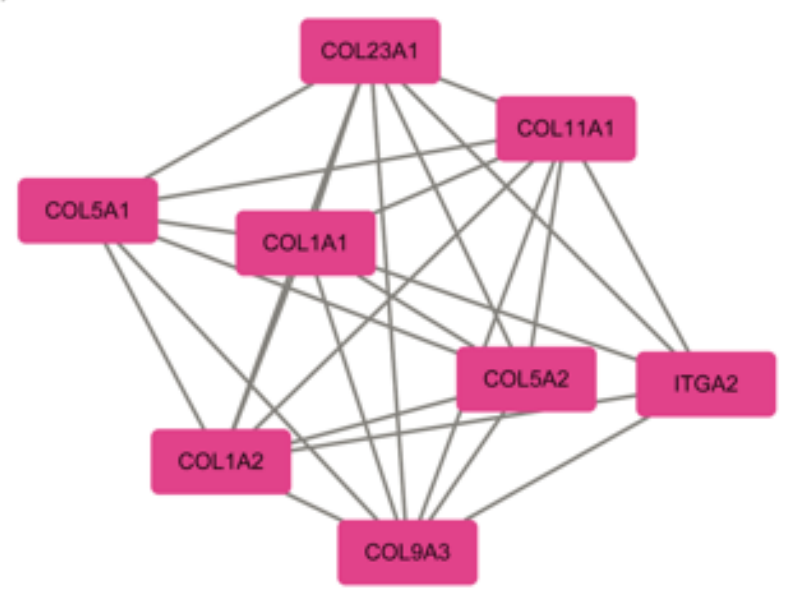

\section{Figure 7}

PPI networks constructed by STRING and modular analysis. (A) DEG PPI network, (B) module 1 of DEGs from PPI network, (C) module 2 of DEGs from PPI network, and (D) module 3 of DEGs from PPI network. Notes: The network consisting of 376 nodes and 361 edges was extracted from the whole protein-protein interaction network. 


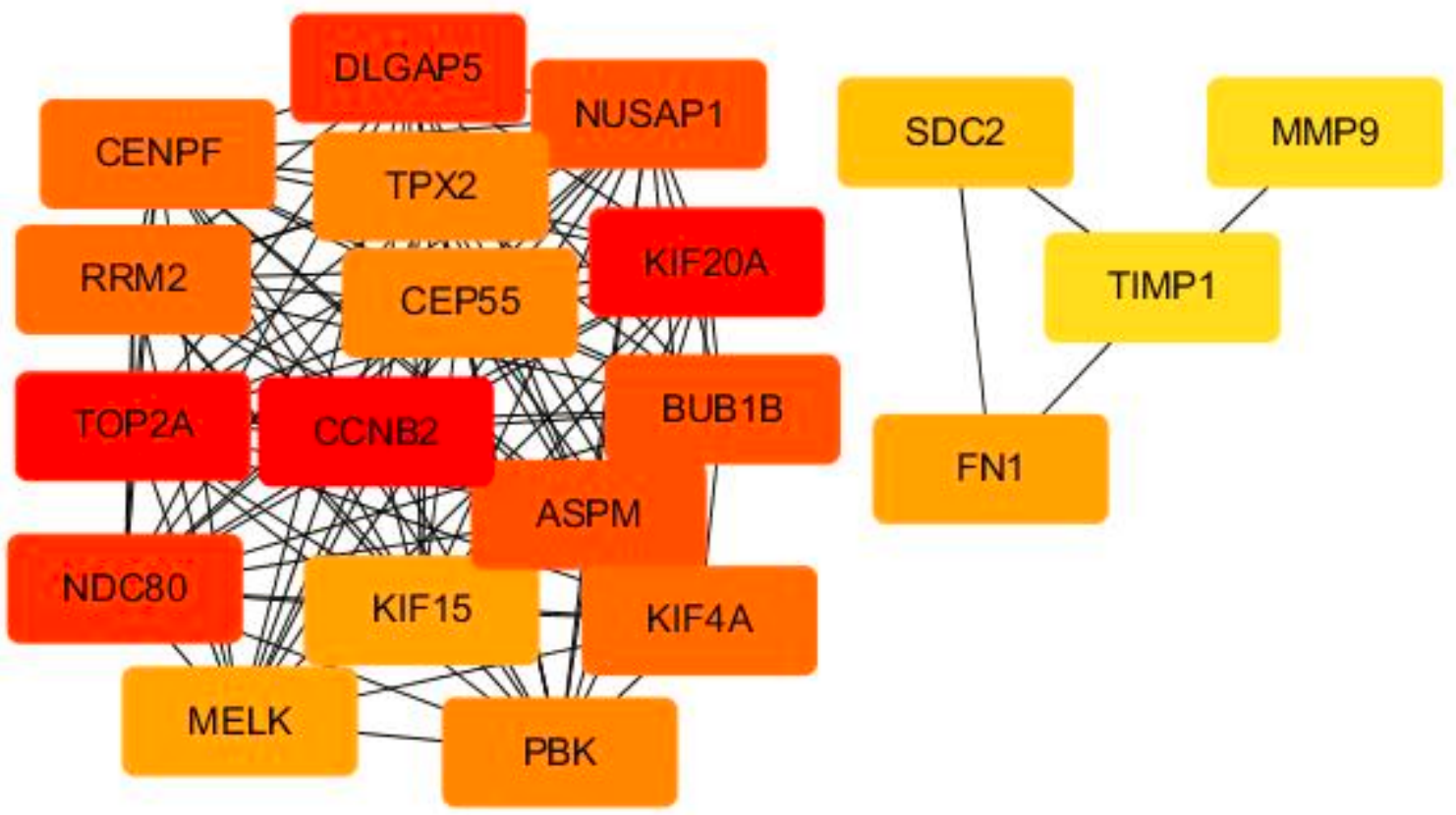

Figure 8

The hub genes formed a network of interactions. 

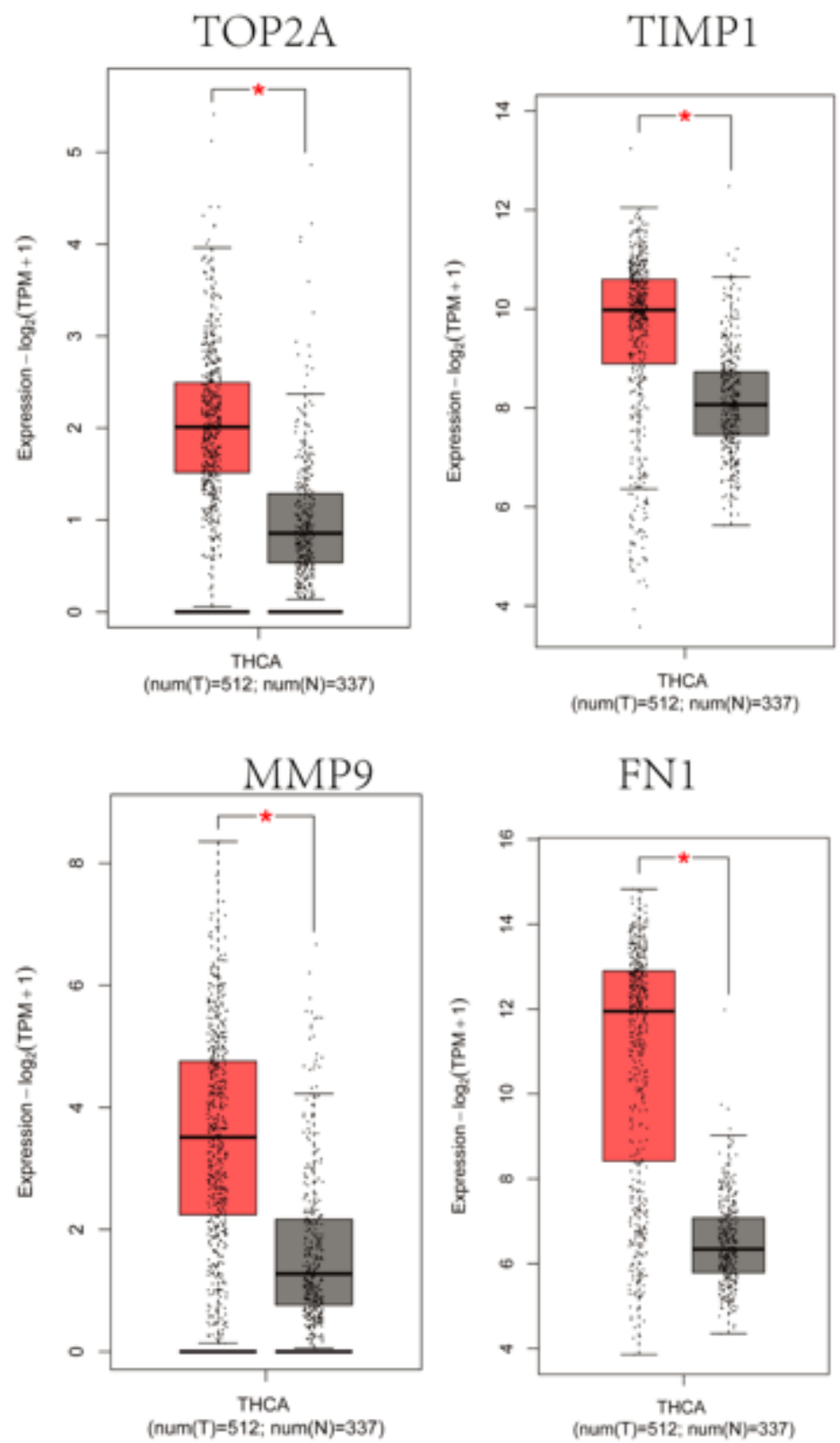

Figure 9

The expression level of hub genes in thyroid cancer tissue through GEPIA. 

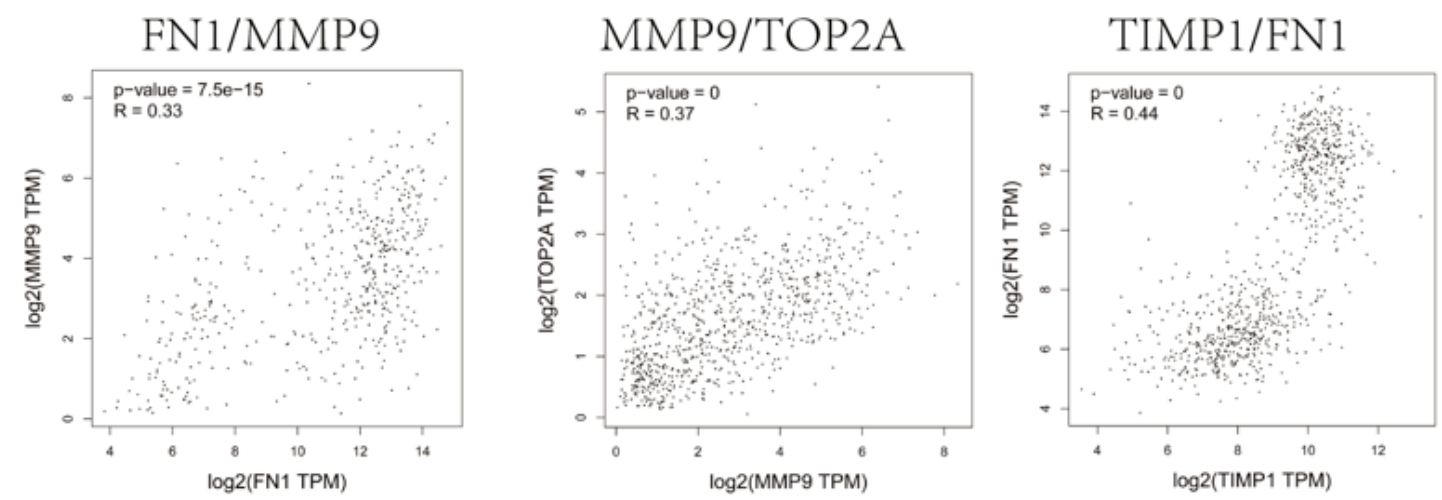

TIMP1/MMP9
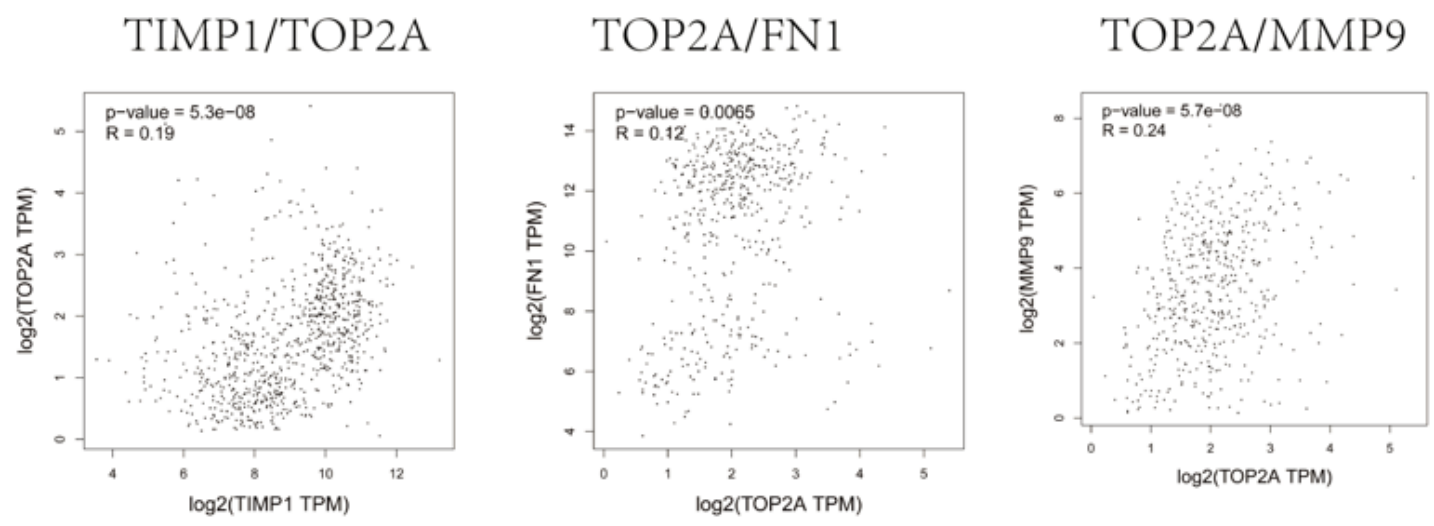

Figure 10

Correlation between hub genes.
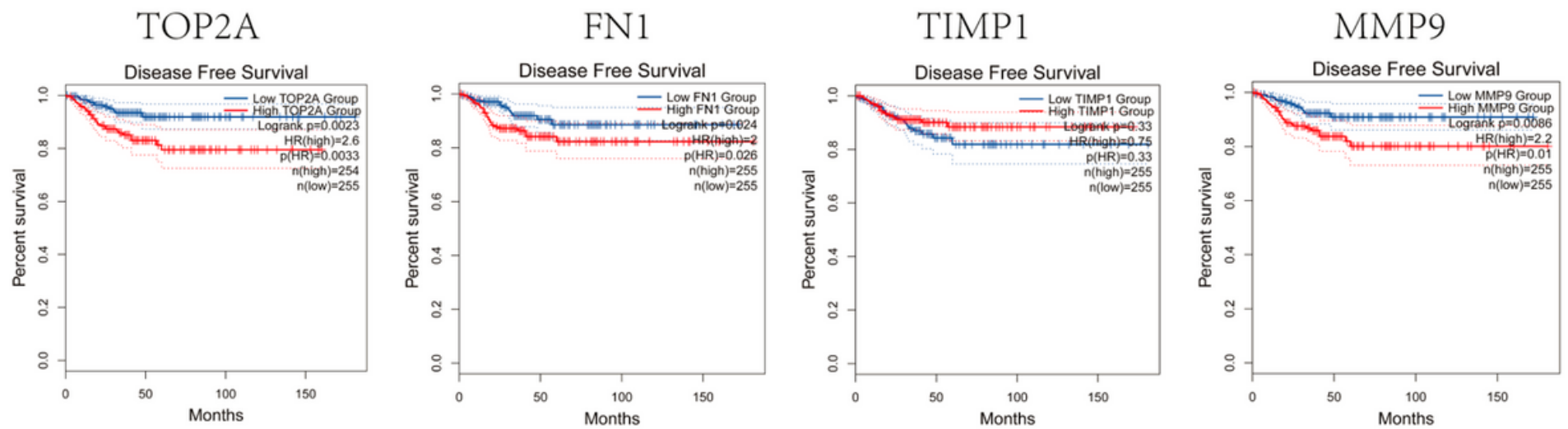

Figure 11

The relationship between hub gene and DFS. 
TOP2A

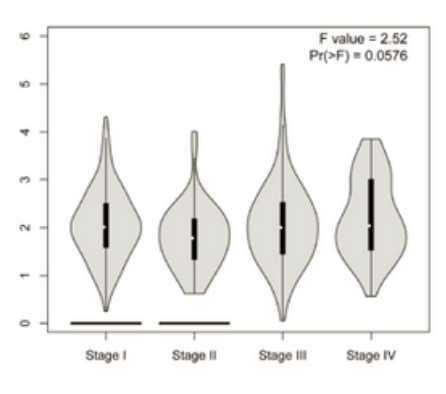

FN1

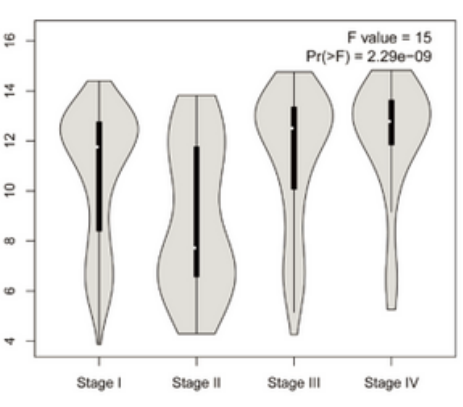

TIMP1

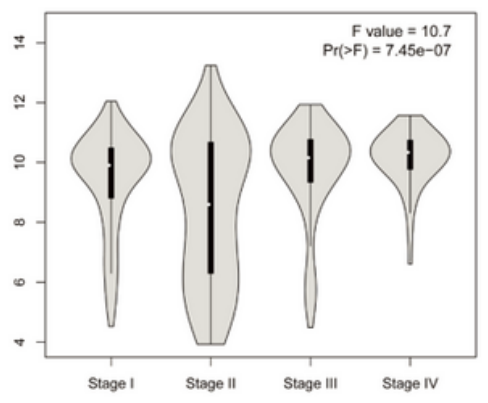

MMP9

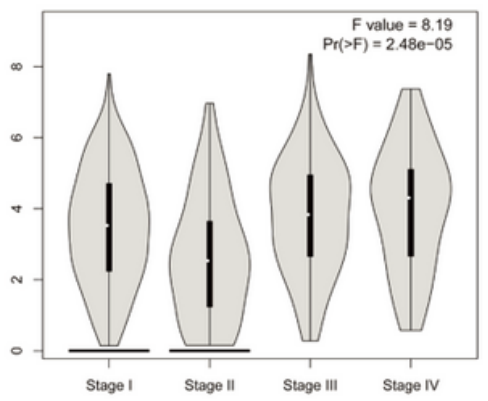

Figure 12

The relationship between hub gene and clinical stage.
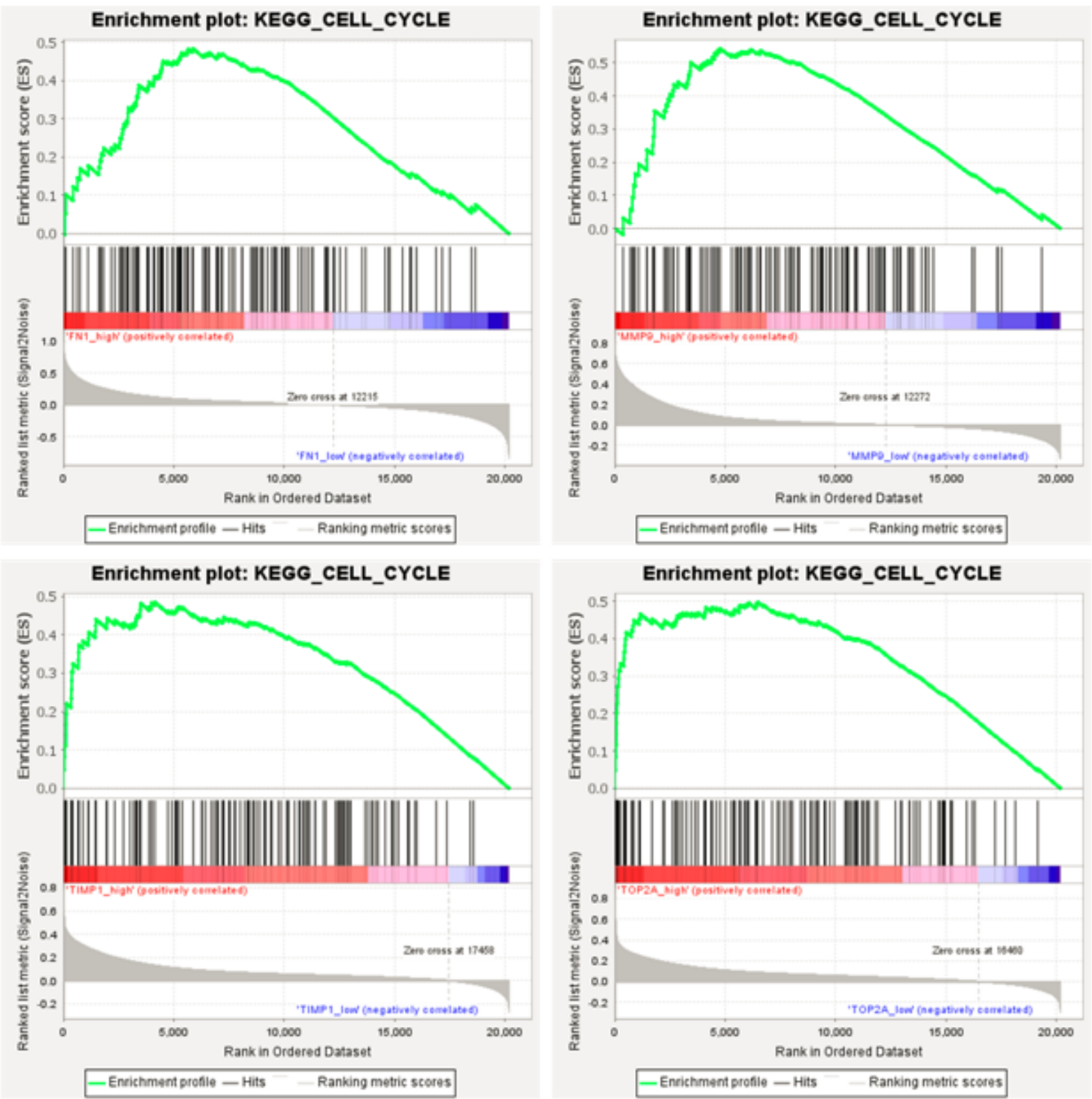
Figure 13

FN1, TOP2A and MMP9 enriched the "cell cycle".

FN1

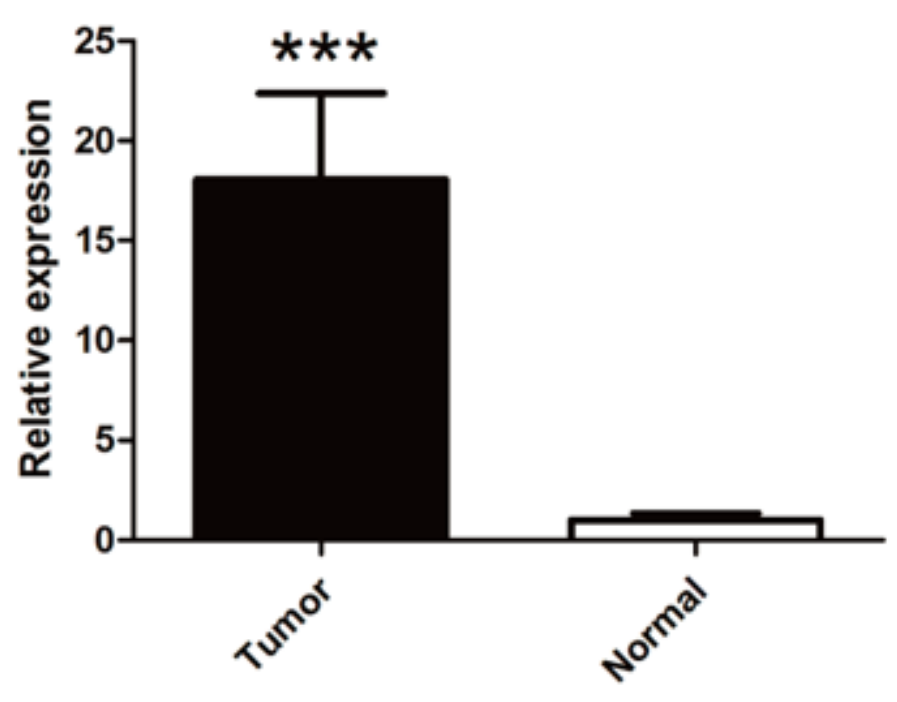

TOP2A

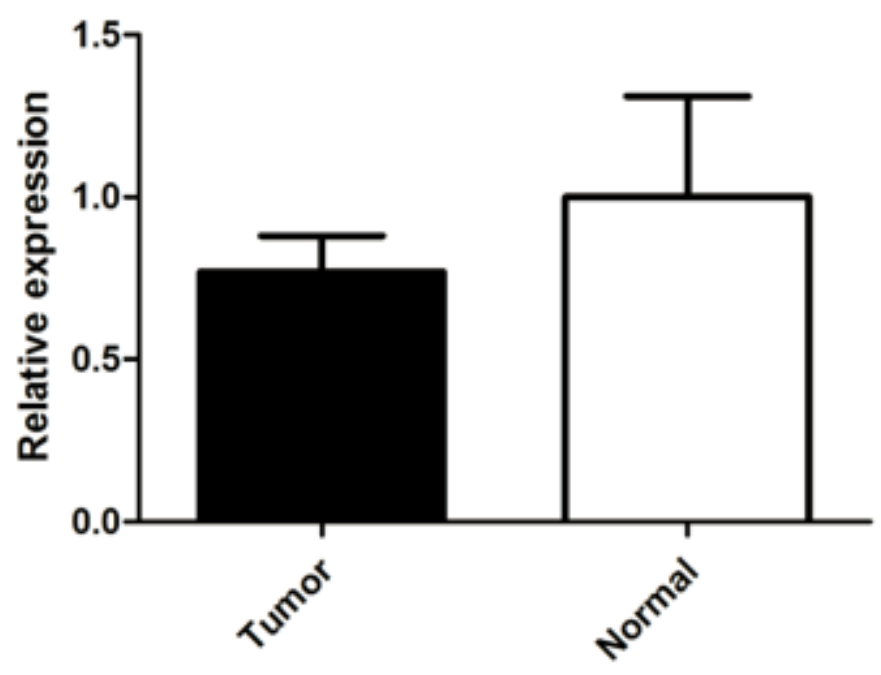

TIMP1

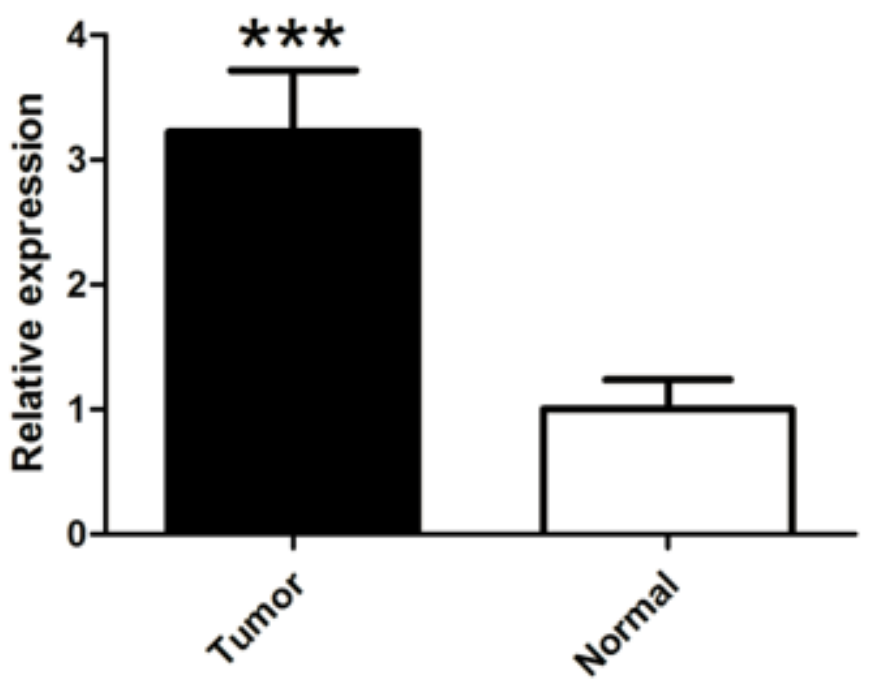

MMP9

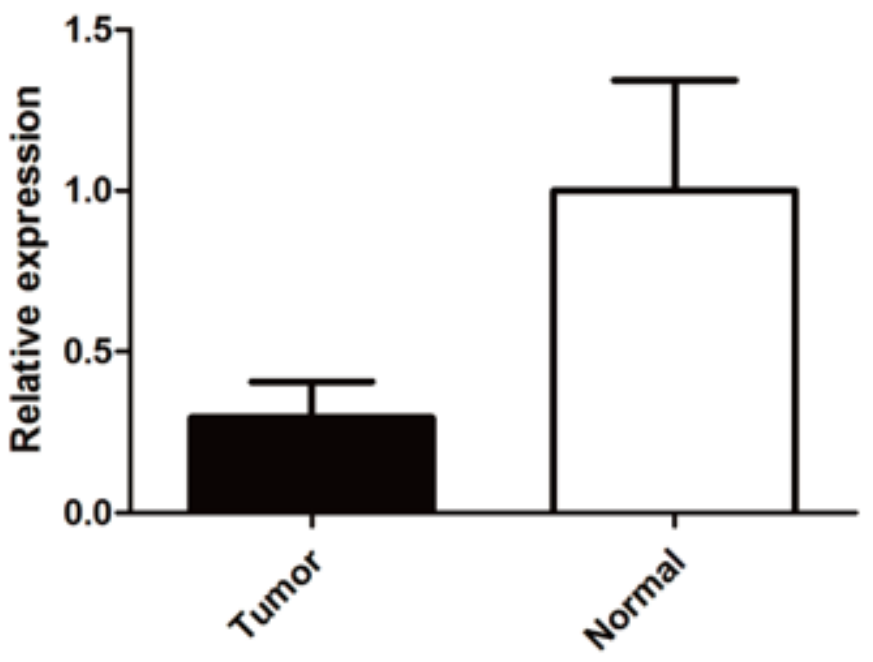

Figure 14

The expression level of hub gene in thyroid cancer tissue. 